\title{
NON-HYPERBOLIC ITERATED FUNCTION SYSTEMS: SEMIFRACTALS AND THE CHAOS GAME
}

\author{
LORENZO J. DÍAZ AND EDGAR MATIAS
}

\begin{abstract}
We consider iterated functions systems (IFS) on compact metric spaces and introduce the concept of target sets. Such sets have very rich dynamical properties and play a similar role as semifractals introduced by Lasota and Myjak do for regular IFSs. We study sufficient conditions which guarantee that the closure of the target set is a local attractor for the IFS. As a corollary, we establish necessary and sufficient conditions for the IFS having a global attractor. We give an example of a non-regular IFS whose target set is nonempty, showing that our approach gives rise to a "new class" of semifractals. Finally, we show that random orbits generated by IFSs draws target sets that are "stable".
\end{abstract}

\section{INTRODUCTION}

Consider finitely many contractions $f_{1}, \ldots, f_{k}$ defined on a complete metric space $X$ and a sequence of independent and identically distributed (i.i.d.) random variables $\mathbb{X}_{n}$ with range $\{1, \ldots, k\}$. It is well known that for every $x$, with probability 1 , the tail sequence of sets $\left\{f_{\mathbb{X}_{n-1}} \circ \cdots \circ f_{\mathbb{X}_{0}}(x): n \geq \ell\right\}_{\ell}$ converges to the unique compact set $K$ satisfying

$$
K=\bigcup_{i=1}^{k} f_{i}(K),
$$

called the Hutchinson attractor. The existence and uniqueness of the latter was proved in 13 .

In this paper, we consider a setting where the maps $f_{1}, \ldots, f_{k}$ are just continuous maps defined on a compact metric space and we translate several results of the so-called "Hutchinson theory", as the ones described above, to this non-contracting setting. The finite family of continuous maps $f_{1}, \ldots, f_{k}$ is called an iterated function system (in what follows, an IFS) and denoted by $\operatorname{IFS}\left(f_{1}, \ldots, f_{k}\right)$. An IFS is called hyperbolic if all of its maps are (uniform) contractions, and non-hyperbolic otherwise.

A key ingredient in this study is the so-called Barnsley-Hutchinson operator (in the sequel, the $\mathrm{BH}$-operator for short). Considering the hyperspace $\mathscr{H}(X)$ of nonempty compact subsets of $X$ endowed with the Hausdorff distance, the BH-operator

2000 Mathematics Subject Classification. 37C70, 28A80, 47H10.

Key words and phrases. Barnsley-Hutchinson operator, chaos game, Conley and strict attractors, iterated function system, semifractals.

This paper is part of the PhD thesis of EM (PUC-Rio) supported by CAPES. LJD is partially supported by CNPq and CNE-Faperj. The authors warmly thank P. Barrientos and K. Gelfert for their useful comments on this paper. 
associated to $\operatorname{IFS}\left(f_{1}, \ldots, f_{k}\right)$ is defined by

$$
\mathcal{B}: \mathscr{H}(X) \rightarrow \mathscr{H}(X), \quad \mathcal{B}(A) \stackrel{\text { def }}{=} \bigcup_{i=1}^{k} f_{i}(A) .
$$

An important aspect of the Hutchinson theory concerns the study of the dynamics of the BH-operator and its fixed points. The Hutchinson attractor of a hyperbolic IFS is the unique fixed point of the BH-operator. Moreover, in this case, this attractor is global, see [13, 2].

A first important class of non-hyperbolic IFSs considered in this study is the one of contracting on average IFSs, see [3]. For this class, when studying IFSs from the probabilistic point of view, the results in [3] fully recovered the results about stationary measures in [13]. On the other hand, from the topological point of view, the scenario may change substantially. The dynamics of the BH-operator of a contracting on average IFS is, in general, very different from the one in the hyperbolic case. For instance, concerning compact fixed points, it may have several ones or none at all. Nevertheless, by [15], it always possesses one (non-necessarily compact) fixed point with very rich dynamical properties which is called its semifractal and, in a certain sense, generalizes the Hutchinson attractor. We observe that semifractals were introduced for so-called regular IFSs (see Section 2.1 for the precise definition), which is a class of IFSs much more general than the contracting on average one. A semifractal is characterised by the following two properties:

(SF1) it is the minimum fixed point of the BH-operator (i.e., it is contained in any other fixed point of the BH-operator) and

(SF2) it attracts each of its compact subsets under the action of the BH-operator.

Another class of non-hyperbolic IFSs was considered by Edalat in [12], also fully recovering aspects of the Hutchinson theory. In what follows, we assume that $X$ is compact. The $\operatorname{IFS}\left(f_{1}, \ldots, f_{k}\right)$ is called weakly hyperbolic if it satisfies the following topological condition:

$$
\lim _{n \rightarrow \infty} \operatorname{diam}\left(f_{\omega_{0}} \circ \cdots \circ f_{\omega_{n}}(X)\right)=0
$$

for every $\omega=\omega_{0} \omega_{1} \omega_{2} \cdots \in \Sigma_{k} \stackrel{\text { def }}{=}\{1, \ldots, k\}^{\mathbb{N}}$. In [12] it is proved that the BHoperator of a weakly hyperbolic IFS has a global attracting fixed point. Note that every hyperbolic IFS is weakly hyperbolic. Observe that by a recent result in [1, after a change of metric, every weakly hyperbolic system can be made hyperbolic.

Bearing the discussion above in mind, we introduce the subset $S_{\mathrm{wh}} \subset \Sigma_{k}$ of weakly hyperbolic sequences defined by

$$
S_{\mathrm{wh}} \stackrel{\text { def }}{=}\left\{\omega \in \Sigma_{k}: \lim _{n \rightarrow \infty} \operatorname{diam}\left(f_{\omega_{0}} \circ \cdots \circ f_{\omega_{n}}(X)\right)=0\right\} .
$$

Note that if the IFS is weakly hyperbolic then $S_{\mathrm{wh}}=\Sigma_{k}$. In this paper, we will recover parts of the Hutchinson theory in "truly" non-hyperbolic settings where we only require that the set $S_{\mathrm{wh}}$ is non-empty. In this analysis, a key object is the so-called target set defined as follows. Associated to the set $S_{\text {wh }}$ we consider the coding map $\pi: S_{\mathrm{wh}} \rightarrow X$ that projects $S_{\mathrm{wh}}$ into the phase space $X$ defined by

$$
\pi: S_{\mathrm{wh}} \rightarrow X, \quad \pi(\omega) \stackrel{\text { def }}{=} \lim _{n \rightarrow \infty} f_{\omega_{0}} \circ \cdots \circ f_{\omega_{n}}(p),
$$

where $p$ is any point of $X$. By definition of the set $S_{\mathrm{wh}}$, this limit always exists and is independent of $p \in X$. The set of weakly hyperbolic sequences generates the 
target set defined by

$$
A_{\mathrm{tar}} \stackrel{\text { def }}{=} \pi\left(S_{\mathrm{wh}}\right) .
$$

Our results show that the target set encodes crucial aspects of the dynamics of the $\mathrm{BH}$-operator. Let us now briefly discuss these results, the precise statements can be found in Section 2

In what follows we always assume that $S_{\mathrm{wh}} \neq \emptyset$. Under this assumption, we prove that the closure of the target set satisfies the two characteristic properties (SF1) and (SF2) of semifractals, see Theorem 1. We also give an example of a non-regular IFS whose target set is non-empty. This shows that our approach gives rise to a "new class" of semifractals.

Let us observe that, in general, the closure of the target set may fail to be a global attractor. In Theorem 2, we study necessary and sufficient conditions guaranteeing that the closure of the target set is a "local" attractor. As a consequence, we state a sufficient and necessary condition assuring that the BH-operator has a global attractor, generalising Edalat's result [12] mentioned above. Moreover, we show that if the $\mathrm{BH}$-operator has a unique fixed point, then it is necessarily a global attractor, see Theorem 3 .

Finally, we investigate under which conditions a random orbit generated by an IFS draws the target. For a given $\operatorname{IFS}\left(f_{1}, \ldots, f_{k}\right)$, consider a sequence of i.i.d. random variables $\mathbb{X}_{n}$ with range in $\{1, \ldots, k\}$ and for $i \in\{1, \ldots, k\}$ let $p_{i} \stackrel{\text { def }}{=}$ $\operatorname{prob}\left(\mathbb{X}_{n}=i\right)>0$. The procedure of choosing at random $i$ with probability $p_{i}$ and moving from $x$ to $f_{i}(x)$ is an algorithm called the (probabilistic) chaos game. In this way, for each $x \in M$, we get a Markov chain $\mathbb{Z}_{n} \stackrel{\text { def }}{=} f_{\mathbb{X}_{n-1}} \circ \cdots \circ f_{\mathbb{X}_{0}}(x)$ moving through the set $X$. For hyperbolic IFSs, a consequence of standard ergodic theorems (see for instance [8]) is that the tail of the Markov chain $\left\{\mathbb{Z}_{n}: n \geq \ell\right\}_{\ell}$ converges in the Hausdorff distance to the Hutchinson attractor with probability one.

An standard way of taking an i.i.d. sequence with range in $\{1, \ldots, k\}$ is the following. Consider the space $\Sigma_{k}$ as a probability space endowed with the product $\sigma$-algebra and the product measure $\mathbb{P}=\nu^{\mathbb{N}}$, where $\nu$ is the probability measure in $\{1, \ldots, k\}$ given by $\nu(i)=p_{i}$. Then the natural projections $\mathbb{X}_{n}(\omega)=\omega_{n}$ is a sequence of i.i.d. random variables. An advantage of considering $\Sigma_{k}$ as the sample space of the random variables $\mathbb{X}_{n}$ is that we can use the structure of $\Sigma_{k}$ to improve the understanding and results about the chaos game, and also to get more elementary proofs. The results in 4] and Theorem 4 illustrate well these assertions. Let us explain this a bit more precisely.

We show in Theorem 4 that if the closure of the target set is stable (see Section2.3 for the definition) then the subset $\mathcal{D}$ of disjunctive sequences of $\Sigma_{k}$ (see Section 2.3 for details) is such that for every $x \in X$ the sequence of tail sets satisfies

$$
\lim _{\ell \rightarrow \infty}\left\{f_{\omega}^{n}(x): n \geq \ell\right\}_{\ell}=\operatorname{closure}\left(A_{\text {tar }}\right), \quad \text { where } \quad f_{\omega}^{n}(x) \stackrel{\text { def }}{=} f_{\omega_{n-1}} \circ \cdots \circ f_{\omega_{0}}(x),
$$

for every $\omega \in \mathcal{D}$. We observe that it follows from its definition that the set $\mathcal{D}$ does not depend on $x$ and satisfies $\mathbb{P}(\mathcal{D})=1$.

The procedure of choosing $\omega$ in a prescribed set and considering the deterministic orbit $f_{\omega}^{n}(x)$ is called the deterministic chaos game. Notice that in this study there is no stationary process associated, hence it is not possible to use ergodic theorems 
(as in the classical theory) or Markov operators (as in [16, 17]) to prove that deterministic orbits draw the attractor. It is worth mentioning that, remarkably, even in the probabilistic chaos game it is possible to avoid such probabilistic techniques, see for instance [6, 5, 7]. Here Theorem 4 contributes to a series of works about the deterministic chaos game, see 4, 18, 7, the novelties of our result are discussed in Section 2.3

Organisation of the paper. In Section 2 we state precisely the main results in this paper. The theorems are presented in Section 2 and proved in Section 3 , which consists of a section of preliminary general results and four subsections dedicated to prove these theorems. In Section 4, we present some examples illustrating our results and hypotheses.

Standing hypotheses. Throughout this paper, we always consider a complete metric space $(X, d)$, an $\operatorname{IFS}\left(f_{1}, \ldots, f_{k}\right)$ of continuous maps defined on $X$, and its corresponding $\mathrm{BH}$-operator $\mathcal{B}$. Thus, these hypotheses are omitted in the statement of our theorems.

Convergence of compact sets is always considered with respect to the Hausdorff distance $d_{H}$ defined as follows. Given a point $x \in X$ and a set $A \subset X$, the distance between $x$ and $A$ is defined by

$$
d(x, A) \stackrel{\text { def }}{=} \inf \{d(x, a): a \in A\} .
$$

Recall that $\mathscr{H}(X)$ is the set of non-empty compact subsets of $X$. The Hausdorff distance between two compact sets $A, B \in \mathscr{H}(X)$ is defined by

$$
d_{H}(A, B) \stackrel{\text { def }}{=} \max \left\{h_{s}(A, B), h_{s}(B, A)\right\}, \quad \text { where } \quad h_{s}(A, B) \stackrel{\text { def }}{=} \sup _{a \in A} d(a, B) .
$$

We observe that $\left(\mathscr{H}(X), d_{H}\right)$ is a compact metric space, see [2].

\section{MAin Results}

2.1. Target sets and semifractals. Our first result shows that the closure of the target set satisfies the properties (SF1) and (SF2) characterising the semifractals of regular IFSs introduced by Lasota and Myjak in [15]. An IFS $\left(f_{1}, \ldots, f_{k}\right)=\mathfrak{F}$ is said to be regular if there are numbers $1 \leq i_{1}<i_{2}<\cdots<i_{\ell} \leq k$ such that $\operatorname{IFS}\left(f_{i_{1}}, \ldots, f_{i_{\ell}}\right)=\mathfrak{F}^{\prime}$ has a global attractor. The global attractor of $\mathfrak{F}^{\prime}$ is called a nucleus of $\mathfrak{F}$ (an IFS may have several nuclei). It is proved in [15] that the BHoperator of any regular IFS $\mathfrak{F}$ has a fixed point, called a semifractal, satisfying the properties (SF1) and (SF2). In the next theorem, replacing the regularity of the IFS by the condition $S_{\mathrm{wh}} \neq \emptyset$, we recover these properties. Recall the definition of the target set $A_{\mathrm{tar}}$ in (1.5).

Theorem 1. Consider an IFS with $S_{\mathrm{wh}} \neq \emptyset$. Then the following holds:

(1) The set $\overline{A_{\mathrm{tar}}}$ is the minimum fixed point of $\mathcal{B}$.

(2) For every compact set $K \subset \overline{A_{\text {tar }}}$ it holds

$$
\lim _{n \rightarrow \infty} d_{H}\left(\mathcal{B}^{n}(K), \overline{A_{\text {tar }}}\right)=0 .
$$

Remark 2.1. The regularity of the IFS does not imply that $S_{\mathrm{wh}} \neq \emptyset$ and viceversa. Example 4.1 provides an IFS with $S_{\text {wh }} \neq \emptyset$ which is not regular. Hence, our approach provides a "new class" of semifractals. Since minimum fixed points are 
unique, it follows that for regular IFSs with $S_{\text {wh }} \neq \emptyset$ the semifractal (as defined by Lasota and Myjak) is the closure of the target set.

Remark 2.2. Zorn's lemma provides a minimal fixed point for the BH-operator (i.e., a fixed point that does not contain properly another fixed point). However, as mentioned above, minimum fixed points may not exist. Note that, by definition, a minimum fixed point is minimal, but the converse is not true in general. Above we have seen two different contexts leading to minimum fixed points: regularity of the IFS and the existence of some weakly hyperbolic sequence. It is natural to ask for a unifying condition guaranteeing the existence of a minimum fixed point of the BH-operator.

2.2. Attractors of the Barnsley-Hutchinson operator. To state our next results precisely we need the following definition.

Definition 2.3 (Strict and Conley attractors). A compact set $A \subset X$ is a strict attractor of the BH-operator if there is an open neighbourhood $U$ of $A$ such that

$$
\lim _{n \rightarrow \infty} \mathcal{B}^{n}(K)=A \text { for every compact set } K \subset U \text {. }
$$

The basin of attraction of $A$ is the largest open neighbourhood of $A$ for which the above property holds. A strict attractor whose basin of attraction is the whole space is a global attractor.

A compact set $S \subset X$ is a Conley attractor of the BH-operator if there exists an open neighbourhood $U$ of $S$ such that

$$
\lim _{n \rightarrow \infty} \mathcal{B}^{n}(\bar{U})=S .
$$

Remark 2.4. The continuity of the BH-operator implies that Conley and strict attractors are both fixed points of $\mathcal{B}$. Note also that strict attractors are Conley attractors but the converse is not true in general.

The next theorem shows that the target set plays a key role in the study of strict attractors of the BH-operator for IFSs with $S_{\mathrm{wh}} \neq \emptyset$.

Theorem 2. Consider an IFS with $S_{\mathrm{wh}} \neq \emptyset$. Then the following holds:

(1) The BH-operator has at most one strict attractor. Moreover, if such a strict attractor exists then it is equal to $\overline{A_{\text {tar }}}$.

(2) $\overline{A_{\mathrm{tar}}}$ is a Conley attractor if and only if it is a strict attractor.

Let us observe that in 20] Vince obtained a result similar to Theorem 2 for a completely different class of non-hyperbolic IFSs. He shows that an IFS consisting of Möbius maps (Möbius IFSs) has at most one strict attractor and that a compact set is a Conley attractor if and only if it is a strict attractor. Note that for Möbius IFSs the set $S_{\mathrm{wh}}$ is empty.

The next theorem generalises Edalat's results in [12] in two ways: (i) it applies to IFSs which are not weakly hyperbolic, (ii) it provides not only a sufficient condition (as in [12]) but also a necessary condition for the existence of a global attractor. To state the theorem consider the set

$$
X^{*} \stackrel{\text { def }}{=} \bigcap_{n \geq 0} \mathcal{B}^{n}(X)
$$

which is a fixed point of $\mathcal{B}$ (see Lemma 3.1). Note that the set $X^{*}$ is the maximum fixed point of the map $\mathcal{B}$ (i.e., every fixed point of $\mathcal{B}$ is contained in $X^{*}$ ). 
Theorem 3. Consider an IFS with $S_{\mathrm{wh}} \neq \emptyset$. The following three assertions are equivalent:

(1) $\overline{A_{\text {tar }}}=X^{*}$,

(2) the $\mathrm{BH}$-operator has a unique fixed point,

(3) $X^{*}$ is a global attractor of the $\mathrm{BH}$-operator.

The Hutchinson theory, the hyperbolic as well as the non-hyperbolic one, has a topological and a probabilistic point of view. Despite their different nature, these two points of view "talk to each other" through the coding map, which plays a key role in both approaches. Let us illustrate this "talk". Starting with the probabilistic side, it is well known, see Letac [19], that if $\mathbb{P}\left(S_{\mathrm{wh}}\right)=1$, then the distribution ("probabilistic image") of the coding map is an attracting 2 stationary measure of the Markov chain $\omega \mapsto \mathbb{Z}_{n}(\omega) \stackrel{\text { def }}{=} f_{\omega}^{n}(x)$, for every $x$. In other words, if $S_{\text {wh }}$ is big in the probabilistic sense, one of the main results of the hyperbolic Hutchinson theory (uniqueness of stationary measures) is recovered. Considering now the topological side, item (1) of Theorem 3 shows that if the target set (i.e, the topological image of the coding map) is topologically big then the BH-operator has a global attractor. Note that when $\mathbb{P}\left(S_{\mathrm{wh}}\right)=1$, it is clear that $\overline{A_{\mathrm{tar}}}$ is the support of the unique stationary measure $m$, which is given by $m(A)=\mathbb{P}\left(\pi^{-1}(A)\right)$. This discussion leads to the following question:

Question 2.5. Theorem 3 claims that the target set is "big" if and only the BHoperator has a global attractor. On the other hand, Letac's result [19] only provides the sufficient condition if $S_{\mathrm{wh}}$ is "big" then there is an attracting stationary measure. Is the converse true?

Remark 2.6. The name target set for $A_{\text {tar }}$ is justified by the following characterisation

$$
A_{\text {tar }}=\left\{x \in X: \text { there is } \omega \in \Sigma_{k} \text { with }\{x\}=\bigcap_{n} f_{\omega_{0}} \circ \cdots \circ f_{\omega_{n}}(X)\right\} .
$$

This follows observing that $\left\{f_{\omega_{0}} \circ \cdots \circ f_{\omega_{n}}(X)\right\}$ is a sequence of nested compact sets. Let us observe that for weakly hyperbolic IFSs, in [12] it is proved that

$$
X^{*}=\bigcup_{\omega \in \Sigma_{k}} I_{\omega}, \quad \text { where } I_{\omega} \stackrel{\text { def }}{=} \bigcap_{n} f_{\omega_{0}} \circ \cdots \circ f_{\omega_{n}}(X) .
$$

In particular, for weakly hyperbolic IFSs it holds $A_{\mathrm{tar}}=X^{*}$.

It is worth mentioning that there are non-weakly hyperbolic IFSs still satisfying $A_{\mathrm{tar}}=\overline{A_{\mathrm{tar}}}=X^{*}$, see Example 4.3 However, since there are cases where $A_{\mathrm{tar}} \subsetneq$ $\overline{A_{\mathrm{tar}}}=X^{*}$, see Example 4.4 it is indispensable considering the closure of the target set in item (1) of Theorem 3 ,

2.3. The chaos game. We now see how random iterations draw "stable" target sets. For that we need some preliminary definitions. Recall that the shift on $\Sigma_{k}$ is the map $\sigma: \Sigma_{k} \rightarrow \Sigma_{k}$ defined by $(\sigma(\omega))_{n}=\omega_{n+1}$. A sequence of $\Sigma_{k}$ whose orbit is dense in $\Sigma_{k}$, with respect to the shift map, is called disjunctive. We denote by $\mathcal{D}$ the set of disjunctive sequences. Note that, as a consequence of Birkhoff's theorem, for every ergodic measure $\mu$ (with respect to $\sigma$ ) with full support it holds $\mu(\mathcal{D})=1$.

\footnotetext{
${ }^{1}$ Note that the terminology in [19] is different. We translated the results to our terminology.

${ }^{2}$ Under the action of the Markov operator associated with the Markov chain.
} 
A fixed point $K$ of the BH-operator is stable if for every open neighbourhood $V$ of $K$ there is an open neighbourhood $V_{0}$ of $K$ such that

$$
\mathcal{B}^{n}\left(V_{0}\right) \subset V \text { for every } n \geq 0 .
$$

For instance, the set $\overline{A_{\mathrm{tar}}}$ is stable if it is a Conley attractor (but this is not a necessary condition, see Example 4.2) or if all the maps of the IFS are non-expanding (i.e., all maps are Lipschitz with constant less than or equal to 1$) 3$.

Theorem 4. Consider an IFS such that $\overline{A_{\mathrm{tar}}}$ is a stable fixed point of the $\mathrm{BH}-$ operator. Then for every $x \in X$ and every disjunctive sequence $\omega \in \Sigma_{k}$ we have

$$
\lim _{\ell \rightarrow \infty} \overline{\left\{f_{\omega}^{n}(x): n \geq \ell\right\}}=\overline{A_{\text {tar }}} .
$$

We now compare Theorem 4 with some previous results in 4, 17, 18.

In [4] the authors consider an $\operatorname{IFS}\left(f_{1}, \ldots, f_{k}\right)$ having a strict attractor $K$ that is strongly fibred, meaning that for every point $x \in K$ and every neighbourhood $V$ of $x$, there is a finite word $c_{1} \ldots c_{\ell}$ such that $f_{c_{\ell}} \circ \cdots \circ f_{c_{1}}(K) \subset V$. Under the strongly fibred condition, it is proved that for every $x$ in the basin of attraction of $K$ it holds

$$
\lim _{\ell \rightarrow \infty}\left\{f_{\omega}^{n}(x): n \geq \ell\right\}=K
$$

for every disjunctive sequence $\omega$. We observe that the closure of the target set is a fixed point strongly fibred but, in general, it is not a strict attractor, therefore the results in [4 cannot be applied since being stable is a weaker condition than being a strict attractor. Even in the case when $\overline{A_{\operatorname{tar}}}$ is a strict attractor, Theorem 4 provides further information: the deterministic chaos game can start at any point $x$ and not only on the basis of attraction of the set $\overline{A_{\mathrm{tar}}}$.

The deterministic chaos game for well-fibred quasi-attractors is studied in [7]. We refrain to give the precise definition of a well-fibred quasi-attractor. For our goals it is sufficient to say that every strong fibred fixed point of BH-operator is also a wellfibred quasi-attractor. In [7] it is proved that given any well fibred quasi-attractor $K$ then, for every disjunctive sequence $\omega$, the tail sequence $\left\{f_{\omega}^{n}(x): n \geq \ell\right\}_{\ell}$ converges to $K$ for every point $x$ in the set

$$
\mathcal{B}^{*}(K) \stackrel{\text { def }}{=}\left\{x \in X: \bigcap_{m \geq 0} \overline{\bigcup_{n \geq m} \mathcal{B}^{n}(\{x\})}=K\right\} .
$$

Since the closure of the target set is strongly fibred, it is also well-fibred and hence the result in [7] can be applied. In this case, Theorem 4 also provides additional information since, even if $A_{\text {tar }}$ is stable, the set $\mathcal{B}^{*}(K)$ may not be the whole space, see Example 4.2 .

Finally, [18 studies the deterministic chaos game for strict attractors of nonexpanding IFSs. As observed above, in this case the target set is stable (but not necessarily a strict attractor, see Example 4.2) and hence Theorem 4 applies to cases which are not covered by [18].

\footnotetext{
${ }^{3}$ In the literature, these systems are also called non-expansive.
} 


\section{Proofs of the Theorems}

3.1. Preliminary results. We start with two auxiliary results. The first one is an elementary lemma about fixed points of the BH-operator and the second one is an important lemma that will be used in the proofs of Theorems 1 and 2

Lemma 3.1. Consider $A \in \mathscr{H}(X)$ such that $\mathcal{B}(A) \subset A$. Then the set

$$
A^{*} \stackrel{\text { def }}{=} \bigcap_{n \geq 0} \mathcal{B}^{n}(A)
$$

is a fixed point of $\mathcal{B}$. In particular, the set $X^{*}$ is a fixed point of $\mathcal{B}$.

Proof. We need the following well-known result whose proof is included for completeness.

Claim 3.2. Let $\left(L_{n}\right)$ be a sequence of nested compact sets, i.e., with $L_{n+1} \subset L_{n}$. Then

$$
\lim _{n \rightarrow \infty} d_{H}\left(L_{n}, L\right)=0, \quad \text { where } L \stackrel{\text { def }}{=} \bigcap_{n \geq 0} L_{n} .
$$

Proof. The proof is by contradiction. If the claim is false there are $\epsilon>0$ and a subsequence $\left(n_{\ell}\right), n_{\ell} \rightarrow \infty$, such that $d_{H}\left(L_{n_{\ell}}, L\right) \geq \epsilon$ for all $\ell$. Since $L \subset L_{n}$ for every $n$, for each $\ell$ there is a point $p_{\ell} \in L_{n_{\ell}}$ such that $d\left(p_{\ell}, L\right) \geq \epsilon$. By compactness, taking a subsequence if necessary, we can assume that $p_{\ell} \rightarrow p$. As $\left(L_{n}\right)$ is nested it follows that $p \in L$, contradicting that $d\left(p_{\ell}, L\right) \geq \epsilon$ for all $\ell$.

Applying Claim 3.2 to the sequence $L_{n}=\mathcal{B}^{n}(A)$ and using the continuity of $\mathcal{B}$, the lemma follows.

Lemma 3.3. Consider an IFS with $S_{\mathrm{wh}} \neq \emptyset$. Then for every compact set $K$ it holds

$$
\lim _{n \rightarrow \infty} h_{s}\left(\overline{A_{\operatorname{tar}}}, \mathcal{B}^{n}(K)\right)=0 .
$$

Proof. The proof is by contradiction. Assume that there are a compact set $K \in$ $\mathscr{H}(X)$ and a sequence $\left(n_{\ell}\right)$ such that $h_{s}\left(\overline{A_{\text {tar }}}, \mathcal{B}^{n_{\ell}}(K)\right) \geq \epsilon$ for every $\ell$. Note that for each $\ell$ there is $p_{n_{\ell}} \in \overline{A_{\operatorname{tar}}}$ with $d\left(p_{n_{\ell}}, \mathcal{B}^{n_{\ell}}(K)\right) \geq \epsilon$. By compactness, we can assume that $p_{n_{\ell}} \rightarrow p^{*} \in \overline{A_{\text {tar }}}$ and that $\mathcal{B}^{n_{\ell}}(K) \rightarrow \widehat{K}$ for some compact set $\widehat{K}$. We need the following elementary claim whose prove we present for completeness:

Claim 3.4. Consider sequences $\left(A_{n}\right)$ in $\mathscr{H}(X)$ and $\left(p_{n}\right)$ of points in $X$ with $A_{n} \rightarrow$ $A$ and $p_{n} \rightarrow p$ in the Hausdorff distance $d_{H}$. Then

$$
d(p, A)=\lim _{n \rightarrow \infty} d\left(p_{n}, A_{n}\right) .
$$

Proof. We use the following "triangular" inequality: given a point $q$ and two compact sets $A$ and $B$ it holds

$$
d(q, A) \leq d(q, B)+d_{H}(A, B) .
$$

Consider the sequences $\left(A_{n}\right)$ and $\left(p_{n}\right)$ in the claim. Applying twice the "triangular" inequality above we get

$$
d(p, A) \leq d\left(p, p_{n}\right)+d\left(p_{n}, A\right) \leq d\left(p, p_{n}\right)+d\left(p_{n}, A_{n}\right)+d_{H}\left(A_{n}, A\right) .
$$

By hypothesis, $d\left(p, p_{n}\right) \rightarrow 0$ and $d_{H}\left(A_{n}, A\right) \rightarrow 0$. We conclude that

$$
d(p, A) \leq \liminf _{n} d\left(p_{n}, A_{n}\right) .
$$


Applying again twice the "triangular" inequality, we get

$$
d\left(p_{n}, A_{n}\right) \leq d\left(p_{n}, p\right)+d\left(p, A_{n}\right) \leq d\left(p_{n}, p\right)+d(p, A)+d_{H}\left(A, A_{n}\right) .
$$

Arguing as above, this implies that

$$
\limsup _{n} d\left(p_{n}, A_{n}\right) \leq d(p, A) .
$$

Equations (3.2) and (3.3) imply the claim.

It follows from Claim 3.4 that

$$
d\left(p^{*}, \widehat{K}\right) \geq \epsilon
$$

We now derive a contradiction from this inequality. By construction, there is $\ell_{0}$ such that

$$
h_{s}\left(B^{n_{\ell}}(K), \widehat{K}\right)<\frac{\epsilon}{3}, \quad \text { for all } \ell \geq \ell_{0} .
$$

Take $q \in B_{\frac{\epsilon}{3}}\left(p^{*}\right) \cap A_{\mathrm{tar}}$. By the definition of $A_{\operatorname{tar}}$ in (2.1), there is a sequence $\omega=\omega_{0} \omega_{1} \ldots \in S_{\mathrm{wh}}$ such that

$$
\bigcap_{n \geq 0} f_{\omega_{0}} \circ \cdots \circ f_{\omega_{n}}(X)=\{q\} .
$$

Therefore there is $m_{0}$ such that

$$
f_{\omega_{0}} \circ \cdots \circ f_{\omega_{m-1}}(K) \subset f_{\omega_{0}} \circ \cdots \circ f_{\omega_{m-1}}(X) \subset B_{\frac{\epsilon}{3}}\left(p^{*}\right) \quad \text { for every } m \geq m_{0} .
$$

Since $f_{\omega_{0}} \circ \cdots \circ f_{\omega_{m-1}}(K) \subset \mathcal{B}^{m}(K)$, we have

$$
\mathcal{B}^{n_{\ell}}(K) \cap B_{\frac{\epsilon}{3}}\left(p^{*}\right) \neq \emptyset \quad \text { for every } \ell \text { big enough. }
$$

Taking now any $z \in \mathcal{B}^{n_{\ell}}(K) \cap B_{\frac{\epsilon}{3}}\left(p^{*}\right)$ (large $\ell$ ) and using equation (3.5) we have

$$
d\left(p^{*}, \widehat{K}\right) \leq d\left(p^{*}, z\right)+d(z, \widehat{K}) \leq \frac{\epsilon}{3}+\frac{\epsilon}{3}<\epsilon,
$$

contradicting (3.4). This ends the proof of the lemma.

3.2. Proof of Theorem 1. The first item of the theorem follows from the next two assertions that we will prove below:

(1) If $K$ is a non-empty compact fixed point of $\mathcal{B}$, then $\overline{A_{\text {tar }}} \subset K$;

(2) $\overline{A_{\text {tar }}}$ is a fixed point of the BH-operator $\mathcal{B}$.

To prove the first assertion, take any point $p \in A_{\mathrm{tar}}$. It follows from the definition of $A_{\mathrm{tar}}$, see Remark 2.6. that there is a sequence $\omega$ such that

$$
\{p\}=\bigcap_{n \geq 0} f_{\omega_{0}} \circ \cdots \circ f_{\omega_{n}}(X) \supset \bigcap_{n \geq 0} f_{\omega_{0}} \circ \cdots \circ f_{\omega_{n}}(K) .
$$

Since the last intersection is non-empty and contained in $K$, it follows that $p \in K$. As this holds for every point $p \in A_{\text {tar }}$, we have that $A_{\operatorname{tar}} \subset K$. As $K$ is closed this implies that $\overline{A_{\text {tar }}} \subset K$.

It remains to prove the second assertion, $\mathcal{B}\left(\overline{A_{\mathrm{tar}}}\right)=\overline{A_{\mathrm{tar}}}$. Note that for $p$ as in (3.6) and every $i=1, \ldots, k$ it holds

$$
\left\{f_{i}(p)\right\}=\bigcap_{n \geq 0} f_{i} \circ f_{\omega_{0}} \circ \cdots \circ f_{\omega_{n}}(X) .
$$

This implies that $\mathcal{B}\left(A_{\mathrm{tar}}\right) \subset A_{\mathrm{tar}}$. Hence, by continuity of the maps $f_{i}, \mathcal{B}\left(\overline{A_{\mathrm{tar}}}\right) \subset$ $\overline{A_{\mathrm{tar}}}$. By definition of the set $\left(\overline{A_{\mathrm{tar}}}\right)^{*}$ in $(\underline{3.1})$, it is clear that $\left(\overline{A_{\mathrm{tar}}}\right)^{*} \subset \overline{A_{\mathrm{tar}}}$. By 
Lemma 3.1. $\left(\overline{A_{\mathrm{tar}}}\right)^{*}$ is a fixed point of $\mathcal{B}$. Since, by the first assertion, the set $\overline{A_{\mathrm{tar}}}$ is contained in every fixed point of $\mathcal{B}$, it follows that $\overline{A_{\mathrm{tar}}} \subset\left(\overline{A_{\mathrm{tar}}}\right)^{*}$ and hence $\bar{A}_{\mathrm{tar}}=\left(\overline{A_{\mathrm{tar}}}\right)^{*}$.

To prove the second item of the theorem, consider any non-empty compact subset $K \subset \overline{A_{\text {tar }}}$. It follows from (3.6) that $\mathcal{B}(K) \subset \overline{A_{\text {tar }}}$ and hence $\mathcal{B}^{n}(K) \subset \overline{A_{\text {tar }}}$ for every $n \geq 0$. In particular, recalling the definition of $h_{s}$ in (1.6), it follows

$$
\lim _{n \rightarrow \infty} h_{s}\left(\mathcal{B}^{n}(K), \overline{A_{\text {tar }}}\right)=0 .
$$

On the other hand, it follows from Lemma 3.3 that $\lim _{n \rightarrow \infty} h_{s}\left(\overline{A_{\text {tar }}}, \mathcal{B}^{n}(K)\right)=0$ and hence

$$
\lim _{n \rightarrow \infty} d_{H}\left(\mathcal{B}^{n}(K), \overline{A_{\text {tar }}}\right)=0 .
$$

3.3. Proof of Theorem 2. If there are no strict attractors we are done. Otherwise, assume that there is a strict attractor $K$. Since $K$ is a fixed point of $\mathcal{B}$, the first item of Theorem 1 implies that $\overline{A_{\text {tar }}} \subset K$. By definition of a strict attractor, the set $K$ attracts every compact set in a neighbourhood of it, and thus $\overline{A_{\mathrm{tar}}}$ is attracted by $K$. Therefore $\overline{A_{\text {tar }}}=K$, proving the first item.

Since every strict attractor is a Conley attractor, to prove the second item of the theorem it is enough to see that if $\overline{A_{\mathrm{tar}}}$ is a Conley attractor then $\overline{A_{\mathrm{tar}}}$ is a strict attractor. Thus, we now assume that $\overline{A_{\text {tar }}}$ is a Conley attractor and hence it has an open neighbourhood $U$ such that $\lim _{n \rightarrow \infty} \mathcal{B}^{n}(\bar{U})=\overline{A_{\mathrm{tar}}}$. To prove that $\overline{A_{\mathrm{tar}}}$ is a strict attractor it is enough to check that for every compact set $K \in \mathscr{H}(\bar{U})$ it holds $\lim _{n \rightarrow \infty} \mathcal{B}^{n}(K)=\overline{A_{\text {tar }}}$. In other words, we need to see that for every $\epsilon>0$ there is $n_{0} \in \mathbb{N}$ such that for every $n \geq n_{0}$ we have

$$
d_{H}\left(\overline{A_{\text {tar }}}, \mathcal{B}^{n}(K)\right)=\max \left\{h_{s}\left(\overline{A_{\text {tar }}}, \mathcal{B}^{n}(K)\right), h_{s}\left(\mathcal{B}^{n}(K), \overline{A_{\text {tar }}}\right)\right\} \leq \epsilon .
$$

Since $\lim _{n \rightarrow \infty} \mathcal{B}^{n}(\bar{U})=\overline{A_{\text {tar }}}$, there is $n_{0}$ such that for every $n \geq n_{0}$ we have

$$
h_{s}\left(\mathcal{B}^{n}(\bar{U}), \overline{A_{\text {tar }}}\right) \leq \epsilon,
$$

which implies that for every $n \geq n_{0}$,

$$
h_{s}\left(\mathcal{B}^{n}(K), \overline{A_{\text {tar }}}\right) \leq h_{s}\left(\mathcal{B}^{n}(\bar{U}), \overline{A_{\text {tar }}}\right) \leq \epsilon .
$$

Hence, to prove (3.8) it remains to see that $h_{s}\left(\overline{A_{\mathrm{tar}}}, \mathcal{B}^{n}(K)\right) \leq \epsilon$ for every $n$ sufficiently large. This is exactly the content of Lemma 3.3 .

The proof of the theorem is now complete.

Scholium 3.5. If $U$ is a neighbourhood of $\overline{A_{\mathrm{tar}}}$ such that $\lim _{n \rightarrow \infty} \mathcal{B}^{n}(\bar{U})=\overline{A_{\mathrm{tar}}}$, then every compact subset of $\bar{U}$ also satisfies $\lim _{n \rightarrow \infty} \mathcal{B}^{n}(K)=\overline{A_{\text {tar }}}$.

3.4. Proof of Theorem [3. Note that by hypothesis the set $A_{\text {tar }}$ is non-empty. We need to prove the equivalence of the following three assertions:

(1) $\overline{A_{\mathrm{tar}}}=X^{*}$;

(2) the BH-operator has a unique fixed point;

(3) $X^{*}$ is a global attractor (a strict attractor whose basin is the whole space).

The implication $(1) \Rightarrow(2)$ follows from the first item of Theorem 1, which in this case means that "the minimum fixed point $\overline{A_{\mathrm{tar}}}$ and the maximum fixed point $X^{*}$ are the same". The converse implication $(1) \Leftarrow(2)$ follows from the fact that $\overline{A_{\text {tar }}}$ and $X^{*}$ are fixed points of $\mathcal{B}$. Thus we get $(1) \Leftrightarrow(2)$

The implication $(3) \Rightarrow(2)$ is immediate. 
To prove $(1) \Rightarrow(3)$ note that, by Claim 3.2, $X^{*}=\lim _{n \rightarrow \infty} \mathcal{B}^{n}(X)$ and thus $X^{*}$ is a Conley attractor. Since, by hypothesis, $\overline{A_{\mathrm{tar}}}=X^{*}$, we have that $\overline{A_{\mathrm{tar}}}$ is a Conley attractor and hence it follows from Scholium 3.5 that $\overline{A_{\mathrm{tar}}}$ is a strict attractor whose basin is the whole space.

3.5. Proof of Theorem 4, By hypothesis, the set $\overline{A_{\mathrm{tar}}}$ is a stable fixed point of the BH-operator. Fixed a point $x$ and a sequence $\omega$, consider the nested sequences of sets

$$
Y_{\ell}(x, \omega) \stackrel{\text { def }}{=}\left\{f_{\omega}^{n}(x): n \geq \ell\right\} .
$$

In view of Claim [3.2, to prove the theorem it is enough to see that for every disjunctive sequence $\omega$ and every point $x$ it holds

$$
\overline{A_{\mathrm{tar}}}=\bigcap_{\ell \geq 0} \overline{\left\{f_{\omega}^{n}(x): n \geq \ell\right\}}=\bigcap_{\ell \geq 0} \overline{Y_{\ell}(x, \omega)} .
$$

To simplify notation, fixed $\omega$ and $x$ as above, write $Y_{\ell}=Y_{\ell}(x, \omega)$.

Proof of the inclusion " $\subset$ ". Take any point $p \in A_{\text {tar }}$ and fix $\ell \geq 0$. We need to see that for every neighbourhood $V$ of $p$ it holds

$$
V \cap Y_{\ell} \neq \emptyset \text {. }
$$

By definition of $A_{\mathrm{tar}}$, there is an arbitrarily large finite sequence $c_{0} \ldots c_{r}$ such that

$$
f_{c_{r}} \circ \cdots \circ f_{c_{0}}(X) \subset V .
$$

We can assume that $r \geq \ell$. Since $\omega$ has dense orbit there is $m_{1}$ such that

$$
\omega_{m_{1}}=c_{0}, \omega_{m_{1}+1}=c_{1}, \ldots, \omega_{m_{1}+r}=c_{r} .
$$

Therefore, from (3.11) it follows

$$
f_{\omega}^{m_{1}+r+1}(x) \in V .
$$

Since $m_{1}+r \geq \ell$ we have that $f_{\omega}^{m_{1}+r+1}(x) \in Y_{\ell}$ and hence $V \cap Y_{\ell} \neq \emptyset$, proving (3.10) and hence the inclusion " $\subset$ ".

Proof of the inclusion " $\supset$ ". Take any neighbourhood $V$ of $\overline{A_{\mathrm{tar}}}$. Since $\overline{A_{\mathrm{tar}}}$ is stable it has a neighbourhood $V_{0} \subset V$ such that $\mathcal{B}^{n}\left(V_{0}\right) \subset V$ for every $n \geq 0$. By definition of $A_{\mathrm{tar}}$, there is a sequence $c_{0} \ldots c_{r}$ with $f_{c_{r}} \circ \cdots \circ f_{c_{0}}(X) \subset V_{0}$. As above, since $\omega$ is disjunctive, there is $m_{1}$ with $\omega_{m_{1}}=c_{0}, \omega_{m_{1}+1}=c_{1}, \ldots, \omega_{m_{1}+r}=c_{r}$. Taking $n_{0}=m_{1}+r$, this implies that $f_{\omega}^{n_{0}+1}(x) \in V_{0}$. From $\mathcal{B}^{n}\left(V_{0}\right) \subset V$ for every $n \geq 0$ it follows that $Y_{n_{0}} \subset V$, and thus $\overline{Y_{n_{0}}} \subset \bar{V}$. As the sequence of sets $\left(\overline{Y_{\ell}}\right)$ is nested, we have that $\bigcap_{\ell \geq 0} \overline{Y_{\ell}} \subset \bar{V}$. Since this holds for every neighbourhood $V$ of $\overline{A_{\text {tar }}}$, we conclude that

$$
\bigcap_{\ell \geq 0} \overline{Y_{\ell}} \subset \overline{A_{\text {tar }}}
$$

proving the inclusion " $\supset$ ".

We have proved the equality in (3.9) and hence the theorem. 


\section{EXAmples}

In this section, we present some examples that adds a bit more to our understanding on the target set and also illustrate the hypotheses in the theorems. The first example, Example 4.1, is the most important one of this section and shows a non-regular IFS whose target is non-empty. Before going to the details, let us say some general words about our constructions and examples.

Note that requiring the target set to be non-empty is equivalent to assume that $S_{\mathrm{wh}} \neq \emptyset$. To construct an $\operatorname{IFS}\left(f_{1}, \ldots, f_{k}\right)$ defined on a compact metric space $X$ with $S_{\mathrm{wh}} \neq \emptyset$, a natural (and also the simplest) procedure is asking some map to be a contraction, or more generally, asking that for some $\ell \in\{1, \ldots, k\}$, there is $p$ such that

$$
\bigcap_{i \geq 1} f_{\ell}^{i}(X)=\{p\} .
$$

However, since the single map $f_{\ell}$ has a fixed point (the point $p$ ) that is a global attractor, such a hypothesis leads to a regular IFS (recall the definition in Section 2.1). We observe that this is precisely the scenario that one finds when studying contracting on average IFSs on compact metric spaces. In Example 4.1, we show how to construct IFSs with $S_{\mathrm{wh}} \neq \emptyset$ without assuming that one of the maps has a fixed point that is a global attractor as in (4.1). The idea is to use Theorem 4.1 in [11 claiming that for IFSs on compacts subsets of $\mathbb{R}^{m}$ satisfying a simple topological condition it holds $\mathbb{P}\left(S_{\mathrm{wh}}\right)=1$, for every product measure $\mathbb{P}=\nu^{\mathbb{N}}$, where $\nu$ is any probability measure on $\{1, \ldots, k\}$ with $\nu(\{i\})>0$ for every $i$. In particular, for the class of IFSs in [11, Theorem 4.1] it holds $S_{\mathrm{wh}} \neq \emptyset$. In Example 4.1] the IFS is defined on $[0,1]$, but using the same idea we can also perform examples in higher dimensions.

In Example 4.2 we construct an IFS satisfying the hypothesis in Theorem 4 such that the closure of the target set is not a Conley attractor.

We now discuss Examples 4.3 and 4.4 togheter. First recall Remark 2.6 stating that for weakly hyperbolic IFSs the target set is equal to the maximum fixed point, i.e., $A_{\operatorname{tar}}=X^{*}$. Example 4.3 shows that this equality can also happen for nonweakly hyperbolic IFSs. This is an example of a "genuinely" non-hyperbolic IFS whose BH-operator has a global attractor fixed point, recall Theorem 3 However, Example 4.4 shows that the equality $A_{\text {tar }}=X^{*}$ is not a necessary condition for the BH-operator having a global attractor fixed point. In fact, Example 4.4 gives an IFS where $\overline{A_{\text {tar }}}$ coincides with the maximum fixed point but $A_{\text {tar }} \subsetneq \overline{A_{\text {tar }}}$. In other words, in Theorem 3 it is indispensable considering the closure on the target set.

In Examples 4.4 and 4.3 , we consider IFSs consisting of two maps on the interval $[0,1]$. We observe that to construct IFSs where the target set has the properties in these examples is much more easier if we allow the IFS to have more than 2 maps. However, IFSs consisting of two maps whose target sets have the properties described above can be found in the constructions of two well known class of attractors (of a dynamical system), the so-called bony attractors and porcupinelike horseshoes, see [14, 9]. Hence, we also took the opportunity in this section to describe the target set of the IFSs used in the construction of these attractors.

We close this section with the quite simple Example 4.5 showing that, in general, IFSs may not have a minimum fixed point. 
Example 4.1 (A non-regular IFS with $S_{\mathrm{wh}} \neq \emptyset$ ). Consider an $\operatorname{IFS}\left(f_{1}, f_{2}\right)$ such that $f_{1}$ and $f_{2}$ are continuous and injective maps defined on $[0,1]$ satisfying:

(1) $f_{1}$ has exactly two fixed points 0 and 1 , where 0 is a repeller and 1 is an attractor;

(2) $f_{2}$ has exactly three fixed points $p_{1}<p_{2}<p_{3}$, where $p_{1}$ and $p_{3}$ are attractors and $p_{2}$ is a repeller;

(3) $f_{2}([0,1])=[\alpha, \beta] \subset(0,1)$ and $p_{1}<f_{1}\left(p_{1}\right)<\beta$;

(4) there is $c \in\left(\alpha, p_{1}\right)$ such that $f_{1}(c)=f_{2}(c)$ and $f_{1}(x) \neq f_{2}(x)$ for all $x \neq c$.

These conditions are depicted in Figure 1 We claim that:

(i) $\operatorname{IFS}\left(f_{1}, f_{2}\right)$ is non-regular;

(ii) $S_{\mathrm{wh}} \neq \emptyset$.

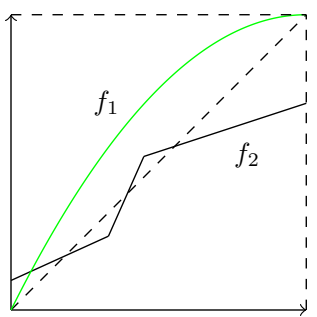

FIGURE 1. A non-regular IFS with $S_{w h} \neq \emptyset$.

Recall first the definition of a regular IFS in Section 2.1. To prove (i), first note that neither $\operatorname{IFS}\left(f_{1}\right)$ or $\operatorname{IFS}\left(f_{2}\right)$ has a global attractor. Thus, it remains to see that $\operatorname{IFS}\left(f_{1}, f_{2}\right)$ has not a global attractor. We prove this assertion by showing that the BH-operator $\mathcal{B}$ associated with $\operatorname{IFS}\left(f_{1}, f_{2}\right)$ has at least two different fixed points (which prevents regularity). Indeed, we have that $[0,1]$ and $\left[p_{1}, 1\right]$ are fixed points of $\mathcal{B}$. It is clear that $[0,1]$ is a fixed point. To see that $\left[p_{1}, 1\right]$ is a fixed point, note that $p_{1}<f_{1}\left(p_{1}\right)<\beta$, hence

$$
\mathcal{B}\left(\left[p_{1}, 1\right]\right)=f_{2}\left(\left[p_{1}, 1\right]\right) \cup f_{1}\left(\left[p_{1}, 1\right]\right)=\left[p_{1}, \beta\right] \cup\left[f_{1}\left(p_{1}\right), 1\right]=\left[p_{1}, 1\right] .
$$

This implies that $\operatorname{IFS}\left(f_{1}, f_{2}\right)$ is non-regular.

To prove (ii), note that 1 is an attracting fixed point of $f_{1}$ and $f_{2}([0,1]) \subset(0,1)$. Hence we get the splitting property $f_{1}^{n} \circ f_{2}([0,1]) \cap f_{2}([0,1])=\emptyset$ for $n$ sufficiently large. Now Theorem 4.1 in [11, implies that $S_{\mathrm{wh}} \neq \emptyset$.

Example 4.2 (An IFS without strict attractors and such that $\overline{A_{\mathrm{tar}}}$ is stable). Consider maps $f_{1}, f_{2}:[0,2] \rightarrow[0,2]$ defined by (see Figure 2):

(1) $f_{1}(x)=\frac{1}{3} x$;

(2) $f_{2}(x)=\frac{1}{3} x+\frac{2}{3}$ for $x \in[0,1]$ and $f_{2}(x)=x$ for $x \in[1,2]$.

We claim that:

(i) $S_{\mathrm{wh}} \neq \emptyset$;

(ii) $\overline{A_{\text {tar }}}$ is not a Conley attractor;

(iii) $\overline{A_{\text {tar }}}$ is stable.

Item (i) follows observing that $f_{1}$ is a contraction.

To prove (ii), consider the auxiliary $\operatorname{IFS}\left(g_{1}, g_{2}\right)$ where $g_{1}=f_{1 \mid[0,1]}$ and $g_{2}=$ $f_{2 \mid[0,1]}$. Let $\mathcal{C}$ be the standard ternary Cantor set in the interval $[0,1]$. We claim that $\overline{A_{\text {tar }}}=\mathcal{C}$. Note that $\mathcal{C}$ is the Hutchinson attractor of $\operatorname{IFS}\left(g_{1}, g_{2}\right)$ (see, for 


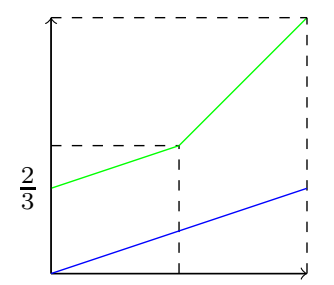

Figure 2. The set $\overline{A_{\text {tar }}}$ is Lyapunov stable and is not a Conley attractor

instance, Example 1 in [13, Section 3.3]). In particular, the set $\mathcal{C}$ is the unique fixed point of the $\mathrm{BH}$-operator $\mathcal{B}$ of $\operatorname{IFS}\left(f_{1}, f_{2}\right)$ contained in $[0,1]$. Since $[0,1]$ is $\mathcal{B}$-invariant, by Lemma 3.1 and minimality (item (1) in Theorem 1) we have $\overline{A_{\operatorname{tar}}} \subset[0,1]^{*} \subset[0,1]$ and therefore $\overline{A_{\text {tar }}}=\mathcal{C}$, proving the claim. To see $\mathcal{C}$ is not a Conley attractor, just note that every open neighbourhood of $\mathcal{C}$ necessarily contains an interval of the form $[1, \delta)$. Since $f_{2}(x)=x$ for all $x \in[1, \delta)$ the assertion follows.

To see (iii), just note that $f_{1}, f_{2}$ are non-expanding maps.

In the next example, we consider the underlying IFS of the bony attractor in 14.

Example 4.3 (A non-weakly hyperbolic IFS on $[0,1]$ with $A_{\text {tar }}=[0,1]$ ). Consider the $\operatorname{IFS}\left(f_{1}, f_{2}\right)$ whose maps $f_{1}$ and $f_{2}$ are defined on $[0,1]$ as follows (see Figure 3):

(1) $f_{1}$ is the piecewise-linear map with "vertices" $(0,0),(0.6,0.2)$, and $(1,0.8)$;

(2) $f_{2}$ is the piecewise-linear map with "vertices" $(0,0.15),(0.4,0.8)$, and $(1,1)$. As observed in [14], these maps have the following properties:

(3) $f_{1} \circ f_{2}$ has a repelling fixed point;

(4) the compositions $f_{1}^{3}, f_{1}^{2} \circ f_{2}, f_{2}^{2} \circ f_{1}$, and $f_{2}^{5}$ are uniform contractions and the union of their images is $[0,1]$.

We claim that:

(i) The $\operatorname{IFS}\left(f_{1}, f_{2}\right)$ is non-weakly hyperbolic;

(ii) $A_{\text {tar }}=[0,1]$.

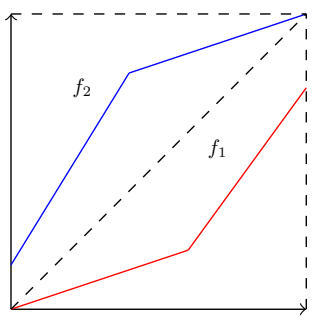

FIgURE 3. The underlying IFS of a bony attractor

The fact that $f_{1} \circ f_{2}$ has a repelling fixed point implies (i): the periodic sequence $\overline{12}$ does not belong to $S_{\text {wh }}$.

To prove (ii), consider the finite set of words corresponding to the compositions in item (4)

$$
W=\{111,112,221,22222\}
$$

and let $E_{W}$ be the subset of $\Sigma_{2}$ consisting of sequences $\omega$ that are a concatenation of words of $W$. It follows from the classical Hutchinson theory for hyperbolic IFSs 
that $E_{W} \subset S_{\text {wh }}$ and $\pi\left(E_{W}\right)=[0,1]$, where $\pi$ is the coding map (this follows from condition(4)). Thus $A_{\mathrm{tar}}=\pi\left(S_{\mathrm{wh}}\right)=[0,1]$.

In the next example we consider the underlying IFS of the porcupine-like horseshoes in [9] and translate the construction in [10, page 12] to our context.

Example 4.4 ( $A_{\mathrm{tar}} \subsetneq \overline{A_{\mathrm{tar}}}$ and $\overline{A_{\mathrm{tar}}}$ is the whole space). Consider the $\operatorname{IFS}\left(f_{1}, f_{2}\right)$ whose maps $f_{1}$ and $f_{2}$ are defined on $[0,1]$ as follows (see Figure 4 ):

(1) $f_{1}(x)=\lambda(1-x), \lambda \in(0,1)$;

(2) $f_{2}$ is an injective continuous map with exactly two fixed points, the repelling fixed point 0 and the attracting fixed point 1 ;

(3) $f_{2}$ is a uniform contraction on $\left[f_{2}^{-1}(\lambda), 1\right]$.

We claim that:

(i) $\overline{A_{\operatorname{tar}}}=[0,1]$;

(ii) $A_{\text {tar }} \subsetneq \overline{A_{\text {tar }}}$.

To prove (i), we start by observing that $\lambda \in \overline{A_{\mathrm{tar}}}$. To see why this is so, take any open neighbourhood $V \subset(0,1)$ of $\lambda$ and note that $f_{1}^{-1}(V)$ is a neighbourhood of 0 . Consider the fixed point $p=\frac{\lambda}{1+\lambda} \in(0,1)$ of $f_{1}$ and note $\overline{1} \in S_{\mathrm{wh}}$ and hence $p \in A_{\mathrm{tar}}$. Since $f_{2}^{n}(p) \rightarrow 1$ as $n \rightarrow \infty$ and $f_{1}(1)=0$, there is $\ell$ such that $f_{1} \circ f_{2}^{\ell}(p) \in f_{1}^{-1}(V)$. Hence $f_{1}^{2} \circ f_{2}^{\ell}(p) \in V$. The invariance of $A_{\operatorname{tar}}$ implies that $A_{\operatorname{tar}} \cap V \neq \emptyset$. Since this holds for every neighbourhood $V$ of $\lambda$ we get $\lambda \in \overline{A_{\mathrm{tar}}}$.

We now prove that $A_{\text {tar }}$ is dense in $[0,1]$ : for every open interval $J \subset(0,1)$ it holds $J \cap A_{\mathrm{tar}} \neq \emptyset$. If $\lambda \in J$ we are done. Otherwise $\lambda \notin J$ and either $J \subset(\lambda, 1] \stackrel{\text { def }}{=} I_{2}$ or $J \subset[0, \lambda) \stackrel{\text { def }}{=} I_{1}$. We claim that

$$
\lambda \in f_{\omega_{m}}^{-1} \circ \cdots \circ f_{\omega_{0}}^{-1}(J), \quad \text { for some } \omega_{0} \ldots \omega_{m} .
$$

Since $\lambda \in \overline{A_{\operatorname{tar}}} \cap f_{\omega_{m}}^{-1} \circ \cdots \circ f_{\omega_{0}}^{-1}(J)$, the invariance of $A_{\operatorname{tar}}$ implies that $J \cap A_{\operatorname{tar}} \neq \emptyset$. Thus it remains to prove (4.2). For that let $\omega_{0}=i$ if $J \subset I_{i}$ and define recursively $\omega_{\ell+1}=i$ if $f_{\omega_{\ell}}^{-1} \circ \cdots \circ f_{\omega_{0}}^{-1}(J) \subset I_{i}$. Note that if at some step $f_{\omega_{\ell}}^{-1} \circ \cdots \circ f_{\omega_{0}}^{-1}(J)$ is not contained neither in $I_{1}$ nor in $I_{2}$ we have that $\lambda \in f_{\omega_{\ell}}^{-1} \circ \cdots \circ f_{\omega_{0}}^{-1}(J)$ and we are done. As $f_{2}^{-1}$ is a uniform expansion on $I_{2}$ and $f_{1}^{-1}$ is a uniform expansion on $I_{1}$, the size of the intervals $f_{\omega_{\ell}}^{-1} \circ \cdots \circ f_{\omega_{0}}^{-1}(J)$ increases exponentially and the recursion stops after a finitely many steps: there is $m$ such that $\lambda \in f_{\omega_{m}}^{-1} \circ \cdots \circ f_{\omega_{0}}^{-1}(J)$, proving (4.2).

To prove (ii), it is enough to see that $1 \notin A_{\text {tar }}$. This follows noting that $\overline{2} \notin S_{\text {wh }}$ and that $1 \notin f_{\omega_{0}} \circ \cdots \circ f_{\omega_{n}}([0,1])$ for any finite sequence $\omega_{0} \ldots \omega_{n}$ such that $\omega_{i}=1$ for some $i$.

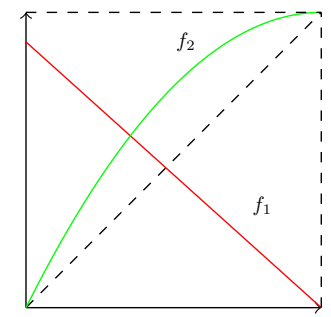

FIgure 4. The underlying IFS of a porcupine-like horseshoe 
Example 4.5 (An IFS without a minimum fixed point). Consider the $\operatorname{IFS}\left(f_{1}, f_{2}\right)$ where $f_{1}, f_{2}:[0,1] \rightarrow[0,1]$ are defined by $f_{1}(x)=x$ and $f_{2}(x)=1-x$. For each $x \in[0,1]$, the set $\{x, 1-x\}$ is a fixed point of $\mathcal{B}$ which is also minimal. Obviously, there is no fixed point contained in all fixed points and hence there is no a minimum fixed point. Note that, in this example, we have $S_{\mathrm{wh}}=\emptyset$.

\section{REFERENCES}

[1] Banakh, T., Kubiś, W. A., Novosad, N., Nowak, M., and Strobin, F. Contractive function systems, their attractors and metrization. Topol. Methods Nonlinear Anal. 46, 2 (2015), 1029-1066.

[2] Barnsley, M. F. Fractals everywhere, second ed. Academic Press Professional, Boston, MA, 1993. Revised with the assistance of and with a foreword by Hawley Rising, III.

[3] Barnsley, M. F., and Elton, J. H. A new class of Markov processes for image encoding. Adv. in Appl. Probab. 20, 1 (1988), 14-32.

[4] Barnsley, M. F., And Leśniak, K. The chaos game on a general iterated function system from a topological point of view. Internat. J. Bifur. Chaos Appl. Sci. Engrg. 24, 11 (2014), 1450139, 10.

[5] Barnsley, M. F., Leśniak, K., And Rypka, M. Chaos game for IFSs on topological spaces. J. Math. Anal. Appl. 435, 2 (2016), 1458-1466.

[6] Barnsley, M. F., And Vince, A. The chaos game on a general iterated function system. Ergodic Theory Dynam. Systems 31, 4 (2011), 1073-1079.

[7] Barrientos, P. G., Ghane, F. H., Malicet, D., and Sarizadeh, A. On the chaos game of iterated function systems. Topol. Methods Nonlinear Anal. 49, 1 (2017), 105-132.

[8] Breiman, L. The strong law of large numbers for a class of Markov chains. Ann. Math. Statist. 31 (1960), 801-803.

[9] Díaz, L. J., And Gelfert, K. Porcupine-like horseshoes: transitivity, Lyapunov spectrum, and phase transitions. Fund. Math. 216, 1 (2012), 55-100.

[10] Díaz, L. J., Gelfert, K., and Rams, M. Almost complete Lyapunov spectrum in step skew-products. Dyn. Syst. 28, 1 (2013), 76-110.

[11] Díaz, L. J., And Matias, E. Stability of the markov operator and synchronization of markovian random products. Nonlinearity 31, 5 (2018), 1782-1806.

[12] Edalat, A. Power domains and iterated function systems. Inform. and Comput. 124, 2 (1996), 182-197.

[13] Hutchinson, J. E. Fractals and self-similarity. Indiana Univ. Math. J. 30, 5 (1981), 713-747.

[14] Kudryashov, Y. G. Bony attractors. Funktsional. Anal. i Prilozhen. 44, 3 (2010), 73-76.

[15] Lasota, A., and Myjak, J. Semifractals. Bull. Polish Acad. Sci. Math. 44, 1 (1996), 5-21.

[16] Lasota, A., and Myjak, J. Fractals, semifractals and Markov operators. Internat. J. Bifur. Chaos Appl. Sci. Engrg. 9, 2 (1999), 307-325. Nonlinearity, bifurcation and chaos: the doors to the future, Part I (Dobieszków, 1996).

[17] Lasota, A., Myjak, J., and Szarek, T. Markov operators and semifractals. In Fractal geometry and stochastics III, vol. 57 of Progr. Probab. Birkhäuser, Basel, 2004, pp. 3-22.

[18] LeŚñAK, K. Random iteration for non-expansive iterated function systems: derandomised algorithm. Int. J. Appl. Nonlinear Sci. 1, 4 (2014), 360-363.

[19] Letac, G. A contraction principle for certain Markov chains and its applications. In Random matrices and their applications (Brunswick, Maine, 1984), vol. 50 of Contemp. Math. Amer. Math. Soc., Providence, RI, 1986, pp. 263-273.

[20] Vince, A. Möbius iterated function systems. Trans. Amer. Math. Soc. 365, 1 (2013), 491509.

Departamento de Matemática PUC-Rio, Marquês de São Vicente 225, Gávea, Rio DE Janeiro 22451-900, Brazil

E-mail address: lodiaz@mat.puc-rio.br

Departamento de Matemática, ICMC-USP São Carlos-SP, Brasil

E-mail address: edgarmatias9271@gmail.com 